\title{
Sensitive and Scalable Online Evaluation with Theoretical Guarantees
}

\author{
Harrie Oosterhuis \\ University of Amsterdam \\ Amsterdam, The Netherlands \\ oosterhuis@uva.nl
}

\author{
Maarten de Rijke \\ University of Amsterdam \\ Amsterdam, The Netherlands \\ derijke@uva.nl
}

\begin{abstract}
Multileaved comparison methods generalize interleaved comparison methods to provide a scalable approach for comparing ranking systems based on regular user interactions. Such methods enable the increasingly rapid research and development of search engines. However, existing multileaved comparison methods that provide reliable outcomes do so by degrading the user experience during evaluation. Conversely, current multileaved comparison methods that maintain the user experience cannot guarantee correctness. Our contribution is two-fold. First, we propose a theoretical framework for systematically comparing multileaved comparison methods using the notions of considerateness, which concerns maintaining the user experience, and fidelity, which concerns reliable correct outcomes. Second, we introduce a novel multileaved comparison method, Pairwise Preference Multileaving (PPM), that performs comparisons based on document-pair preferences, and prove that it is considerate and has fidelity. We show empirically that, compared to previous multileaved comparison methods, PPM is more sensitive to user preferences and scalable with the number of rankers being compared.
\end{abstract}

\section{INTRODUCTION}

Evaluation is of tremendous importance to the development of modern search engines. Any proposed change to the system should be verified to ensure it is a true improvement. Online approaches to evaluation aim to measure the actual utility of an Information Retrieval (IR) system in a natural usage environment [14]. Interleaved comparison methods are a within-subject setup for online experimentation in IR. For interleaved comparison, two experimental conditions ("control" and "treatment") are typical. Recently, multileaved comparisons have been introduced for the purpose of efficiently comparing large numbers of rankers [2,27]. These multileaved comparison methods were introduced as an extension to interleaving and the majority are directly derived from their interleaving counterparts [27, 28]. The effectiveness of these methods has thus far only been measured using simulated experiments on public datasets. While this gives some insight into the general sensitivity of a method, there is no work that assesses under what

Permission to make digital or hard copies of all or part of this work for personal or classroom use is granted without fee provided that copies are not made or distributed for profit or commercial advantage and that copies bear this notice and the full citation on the first page. Copyrights for components of this work owned by others than the author(s) must be honored. Abstracting with credit is permitted. To copy otherwise, or republish, to post on servers or to redistribute to lists, requires prior specific permission and/or a fee. Request permissions from permissions@acm.org.

CIKM'17, November 6-10, 2017, Singapore.

() 2017 Copyright held by the owner/author(s). Publication rights licensed to ACM. ISBN $978-1-4503-4918-5 / 17 / 11 \ldots \$ 15.00$

DOI: https://doi.org/10.1145/3132847.3132895 circumstances these methods provide correct outcomes and when they break. Without knowledge of the theoretical properties of multileaved comparison methods we are unable to identify when their outcomes are reliable.

In prior work on interleaved comparison methods a theoretical framework has been introduced that provides explicit requirements that an interleaved comparison method should satisfy [13]. We take this approach as our starting point and adapt and extend it to the setting of multileaved comparison methods. Specifically, the notion of fidelity is central to Hofmann et al. [13]'s previous work; Section 3 describes the framework with its requirements of fidelity. In the setting of multileaved comparison methods, this means that a multileaved comparison method should always recognize an unambiguous winner of a comparison. We also introduce a second notion, considerateness, which says that a comparison method should not degrade the user experience, e.g., by allowing all possible permutations of documents to be shown to the user. In this paper we examine all existing multileaved comparison methods and find that none satisfy both the considerateness and fidelity requirements. In other words, no existing multileaved comparison method is correct without sacrificing the user experience.

To address this gap, we propose a novel multileaved comparison method, Pairwise Preference Multileaving (PPM). PPM differs from existing multileaved comparison methods as its comparisons are based on inferred pairwise document preferences, whereas existing multileaved comparison methods either use some form of document assignment $[27,28]$ or click credit functions [2,27]. We prove that PPM meets both the considerateness and the fidelity requirements, thus PPM guarantees correct winners in unambiguous cases while maintaining the user experience at all times. Furthermore, we show empirically that PPM is more sensitive than existing methods, i.e., it makes fewer errors in the preferences it finds. Finally, unlike other multileaved comparison methods, PPM is computationally efficient and scalable, meaning that it maintains most of its sensitivity as the number of rankers in a comparison increases.

In this paper we address the following research questions:

RQ1 Does PPM meet the fidelity and considerateness requirements?

RQ2 Is PPM more sensitive than existing methods when comparing multiple rankers?

To summarize, our contributions are:

(1) A theoretical framework for comparing multileaved comparison methods;

(2) A comparison of all existing multileaved comparison methods in terms of considerateness, fidelity and sensitivity;

(3) A novel multileaved comparison method that is considerate and has fidelity and is more sensitive than existing methods. 


\section{RELATED WORK}

Evaluation of information retrieval systems is a core problem in IR. Two types of approach are common to designing reliable methods for measuring an IR system's effectiveness. Offline approaches such as the Cranfield paradigm [26] are effective for measuring topical relevance, but have difficulty taking into account contextual information including the user's current situation, fast changing information needs, and past interaction history with the system [14] In contrast, online approaches to evaluation aim to measure the actual utility of an IR system in a natural usage environment. User feedback in online evaluation is usually implicit, in the form of clicks, dwell time, etc.

By far the most common type of controlled experiment on the web is $A / B$ testing $[19,20]$. This is a classic between-subject experiment, where each subject is exposed to one of two conditions, control-the current system-and treatment-an experimental system that is assumed to outperform the control.

An alternative experiment design uses a within-subject setup, where all study participants are exposed to both experimental conditions. Interleaved comparisons [15, 25] have been developed specifically for online experimentation in IR. Interleaved comparison methods have two main ingredients. First, a method for constructing interleaved result lists specifies how to select documents from the original rankings ("control" and "treatment"). Second, a method for inferring comparison outcomes based on observed user interactions with the interleaved result list. Because of their withinsubject nature, interleaved comparisons can be up to two orders of magnitude more efficient than $\mathrm{A} / \mathrm{B}$ tests in effective sample size for studies of comparable dependent variables [4].

For interleaved comparisons, two experimental conditions are typical. Extensions to multiple conditions have been introduced by Schuth et al. [27]. Such multileaved comparisons are an efficient online evaluation method for comparing multiple rankers simultaneously. Similar to interleaved comparison methods [12, 17, 24, 25], a multileaved comparison infers preferences between rankers. Interleaved comparisons do this by presenting users with interleaved result lists; these represent two rankers in such a way that a preference between the two can be inferred from clicks on their documents. Similarly, for multileaved comparisons multileaved result lists are created that allow more than two rankers to be represented in the result list. As a consequence, multileaved comparisons can infer preferences between multiple rankers from a single click. Due to this property multileaved comparisons require far fewer interactions than interleaved comparisons to achieve the same accuracy when multiple rankers are involved [27, 28].

The general approach for every multileaved comparison method is described in Algorithm 1; here, a comparison of a set of rankers $\mathcal{R}$ is performed over $T$ user interactions. After the user submits a query $q$ to the system (Line 4 ), a ranking $l_{i}$ is generated for each ranker $\mathbf{r}_{i}$ in $\mathcal{R}$ (Line 6). These rankings are then combined into a single result list by the multileaving method (Line 7); we refer to the resulting list $\mathbf{m}$ as the multileaved result list. In theory a multileaved result list could contain the entire document set, however in practice a length $k$ is chosen beforehand, since users generally only view a restricted number of result pages. This multileaved result list is presented to the user who has the choice to interact with it or

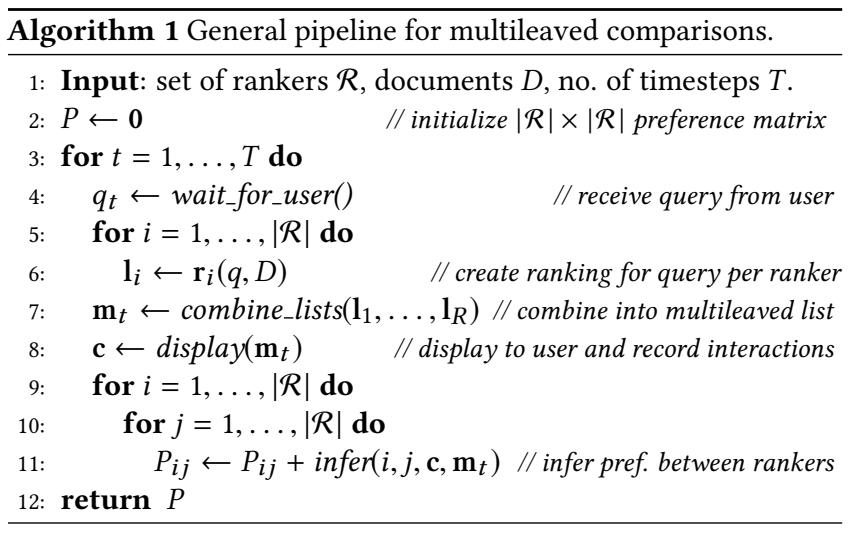

not. Any interactions are recorded in $\mathbf{c}$ and returned to the system (Line 8). While c could contain any interaction information [18], in practice multileaved comparison methods only consider clicks. Preferences between the rankers in $\mathcal{R}$ can be inferred from the interactions and the preference matrix $P$ is updated accordingly (Line 11). The method of inference (Line 11) is defined by the multileaved comparison method (Line 7). By aggregating the inferred preferences of many interactions a multileaved comparison method can detect preferences of users between the rankers in $\mathcal{R}$. Thus it provides a method of evaluation without requiring a form of explicit annotation.

By instantiating the general pipeline for multileaved comparisons shown in Algorithm 1, i.e., the combination method at Line 6 and the inference method at Line 11, we obtain a specific multileaved comparison method. We detail all known multileaved comparison methods in Section 4 below.

What we add on top of the work discussed above is a theoretical framework that allows us to assess and compare multileaved comparison methods. In addition, we propose an accurate and scalable multileaved comparison method that is the only one to satisfy the properties specified in our theoretical framework and that also proves to be the most efficient multileaved comparison method in terms of much reduced data requirements.

\section{A FRAMEWORK FOR ASSESSING MULTILEAVED COMPARISON METHODS}

Before we introduce a novel multileaved comparison method in Section 5, we propose two theoretical requirements for multileaved comparison methods. These theoretical requirements will allow us to assess and compare existing multileaved comparison methods. Specifically, we introduce two theoretical properties: considerateness and fidelity. These properties guarantee correct outcomes in unambigious cases while always maintaining the user experience. In Section 4 we show that no currently available multileaved comparison method satisfies both properties. This motivates the introduction of a method that satisfies both properties in Section 5.

\subsection{Considerateness}

Firstly, one of the most important properties of a multileaved comparison method is how considerate it is. Since evaluation is done online it is important that the search experience is not substantially 
altered [15, 24]. In other words, users should not be obstructed to perform their search tasks during evaluation. As maintaining a user base is at the core of any search engine, methods that potentially degrade the user experience are generally avoided. Therefore, we set the following requirement: the displayed multileaved result list should never show a document $d$ at a rank $i$ if every ranker in $\mathcal{R}$ places it at a lower rank. Writing $r\left(d, \mathbf{l}_{j}\right)$ for the rank of $d$ in the ranking $\mathbf{l}_{j}$ produced by ranker $\mathbf{r}_{j}$, this boils down to:

$$
\mathbf{m}_{i}=d \rightarrow \exists \mathbf{r}_{j} \in \mathcal{R}, r\left(d, \mathbf{l}_{j}\right) \leq i .
$$

Requirement 1 guarantees that a document can never be displayed higher than any ranker would. In addition, it guarantees that if all rankers agree on the top $n$ documents, the resulting multileaved result list $\mathbf{m}$ will display the same top $n$.

\subsection{Fidelity}

Secondly, the preferences inferred by a multileaved comparison method should correspond with those of the user with respect to retrieval quality, and should be robust to user behavior that is unrelated to retrieval quality [15]. In other words, the preferences found should be correct in terms of ranker quality. However, in many cases the relative quality of rankers is unclear. For that reason we will use the notion of fidelity [13] to compare the correctness of a multileaved comparison method. Fidelity was introduced by Hofmann et al. [13] and describes two general cases in which the preference between two rankers is unambiguous. To have fidelity the expected outcome of a method is required to be correct in all matching cases. However, the original notion of fidelity only considers two rankers as it was introduced for interleaved comparison methods, therefore the definition of fidelity must be expanded to the multileaved case. First we describe the following concepts:

Uncorrelated clicks. Clicks are considered uncorrelated if relevance has no influence on the likelihood that a document is clicked. We write $r\left(d_{i}, \mathbf{m}\right)$ for the rank of document $d_{i}$ in multileaved result list $\mathbf{m}$ and $P\left(\mathbf{c}_{l} \mid q, \mathbf{m}_{l}=d_{i}\right)$ for the probability of a click at the rank $l$ at which $d_{i}$ is displayed: $l=r\left(d_{i}, \mathbf{m}\right)$. Then, for a given query $q$

$$
\begin{aligned}
& \operatorname{uncorrelated}(q) \Leftrightarrow \\
& \qquad \forall l, \forall d_{i, j}, P\left(\mathbf{c}_{l} \mid q, \mathbf{m}_{l}=d_{i}\right)=P\left(\mathbf{c}_{l} \mid q, \mathbf{m}_{l}=d_{j}\right) .
\end{aligned}
$$

Correlated clicks. We consider clicks correlated if there is a positive correlation between document relevance and clicks. However we differ from Hofmann et al. [13] by introducing a variable $k$ that denotes at which rank users stop considering documents. Writing $P\left(\mathbf{c}_{i} \mid \operatorname{rel}\left(\mathbf{m}_{i}, q\right)\right)$ for the probability of a click at rank $i$ if a document relevant to query $q$ is displayed at this rank, we set

$$
\begin{aligned}
\operatorname{correlated}(q, k) \Leftrightarrow \forall i \geq k, P\left(\mathbf{c}_{i}\right) & =0 \\
\wedge \forall i<k, P\left(\mathbf{c}_{i} \mid \operatorname{rel}\left(\mathbf{m}_{i}, q\right)\right) & >P\left(\mathbf{c}_{i} \mid \neg \operatorname{rel}\left(\mathbf{m}_{i}, q\right)\right) .
\end{aligned}
$$

Thus under correlated clicks a relevant document is more likely to be clicked than a non-relevant one at the same rank, if they appear above rank $k$.

Pareto domination. Ranker $\mathbf{r}_{1}$ Pareto dominates ranker $\mathbf{r}_{2}$ if all relevant documents are ranked at least as high by $\mathbf{r}_{1}$ as by $\mathbf{r}_{2}$ and $\mathbf{r}_{1}$ ranks at least one relevant document higher. Writing rel for the set of relevant documents that are ranked above $k$ by at least one ranker, i.e., $r e l=\left\{d \mid \operatorname{rel}(d, q) \wedge \exists \mathbf{r}_{n} \in \mathcal{R}, r\left(d, \mathbf{l}_{n}\right)>k\right\}$, we require that the following holds for every query $q$ and any rank $k$ :

$$
\begin{aligned}
& \operatorname{Pareto}\left(\mathbf{r}_{i}, \mathbf{r}_{j}, q, k\right) \Leftrightarrow \\
& \quad \forall d \in \operatorname{rel}, r\left(d, \mathbf{l}_{i}\right) \leq r\left(d, \mathbf{l}_{j}\right) \wedge \exists d \in r e l, r\left(d, \mathbf{l}_{i}\right)<r\left(d, \mathbf{l}_{j}\right) .
\end{aligned}
$$

Then, fidelity for multileaved comparison methods is defined by the following two requirements:

(1) Under uncorrelated clicks the expected outcome may find no preferences between any two rankers in $\mathcal{R}$ :

$$
\forall q, \forall\left(\mathbf{r}_{i}, \mathbf{r}_{j}\right) \in \mathcal{R} \text {, uncorrelated }(q) \Rightarrow E\left[P_{i j} \mid q\right]=0 .
$$

(2) Under correlated clicks, a ranker that Pareto dominates all other rankers must win the multileaved comparison in expectation:

$$
\begin{aligned}
\forall k, \forall q, \forall \mathbf{r}_{i} \in \mathcal{R},(\operatorname{correlated}(q, k) \wedge \\
\left.\forall \mathbf{r}_{j} \in \mathcal{R}, i \neq j \rightarrow \operatorname{Pareto}\left(\mathbf{r}_{i}, \mathbf{r}_{j}, q, k\right)\right) \\
\Rightarrow\left(\forall \mathbf{r}_{j} \in \mathcal{R}, i \neq j \rightarrow E\left[P_{i j} \mid q\right]>0\right) .
\end{aligned}
$$

Note that for the case where $|\mathcal{R}|=2$ and if only $k=|D|$ is considered, these requirements are the same as for interleaved comparison methods [13]. The $k$ parameter was added to allow for fidelity in considerate methods, since it is impossible to detect preferences at ranks that users never consider without breaking the considerateness requirement. We argue that differences at ranks that users are not expected to observe should not affect comparison outcomes. Fidelity is important for a multileaved comparison method as it ensures that an unambiguous winner is expected to be identified. Additionally, the first requirement ensures unbiasedness when clicks are unaffected by relevancy.

\subsection{Additional properties}

In addition to the two theoretical properties listed above, considerateness and fidelity, we also scrutinize multileaved comparison methods to determine whether they accurately find preferences between all rankers in $\mathcal{R}$ and minimize the number of user impressions required do so. This empirical property is commonly known as sensitivity [13, 27]. In Section 6 we describe experiments that are aimed at comparing the sensitivity of multileaved comparison methods. Here, two aspects of every comparison are considered: the level of error at which a method converges and the number of impressions required to reach that level. Thus, an interleaved comparison method that learns faster initially but does not reach the same final level of error is deemed worse.

\section{AN ASSESSMENT OF EXISTING MULTILEAVED COMPARISON METHODS}

We briefly examine all existing multileaved comparison methods to determine whether they meet the considerateness and fidelity requirements. An investigation of the empirical sensitivity requirement is postponed until Section 6 and 7.

\subsection{Team Draft Multileaving}

Team-Draft Multileaving (TDM) was introduced by Schuth et al. [27] and is based on the previously proposed Team Draft Interleaving (TDI) [25]. Both methods are inspired by how team assignments are often chosen for friendly sport matches. The multileaved result 
list is created by sequentially sampling rankers without replacement; the first sampled ranker places their top document at the first position of the multileaved list. Subsequently, the next sampled ranker adds their top pick of the remaining documents. When all rankers have been sampled, the process is continued by sampling from the entire set of rankers again. The method is stops when all documents have been added. When a document is clicked, TDM assigns the click to the ranker that contributed the document. For each impression binary preferences are inferred by comparing the number of clicks each ranker received.

It is clear that TDM is considerate since each added document is the top pick of at least one ranker. However, TDM does not meet the fidelity requirements. This is unsurprising as previous work has proven that TDI does not meet these requirements [12, 13, 24]. Since TDI is identical to TDM when the number of rankers is $|\mathcal{R}|=2$, TDM does not have fidelity either.

\subsection{Optimized Multileaving}

Optimized Multileaving (OM) was proposed by Schuth et al. [27] and serves as an extension of Optimized Interleaving (OI) introduced by Radlinski and Craswell [24]. The allowed multileaved result lists of OM are created by sampling rankers with replacement at each iteration and adding the top document of the sampled ranker. However, the probability that a multileaved result list is shown is not determined by the generative process. Instead, for a chosen credit function OM performs an optimization that computes a probability for each multileaved result list so that the expected outcome is unbiased and sensitive to correct preferences.

All of the allowed multileaved result lists of OM meet the considerateness requirement, and in theory instantiations of OM could have fidelity. However, in practice OM does not meet the fidelity requirements. There are two main reasons for this. First, it is not guaranteed that a solution exists for the optimization that OM performs. For the interleaving case this was proven empirically when $k=10$ [24]. However, this approach does not scale to any number of rankers. Secondly, unlike OI, OM allows more result lists than can be computed in a feasible amount of time. Consider the top $k$ of all possible multileaved result lists; in the worst case this produces $|\mathcal{R}|^{k}$ lists. Computing all lists for a large value of $|\mathcal{R}|$ and performing linear constraint optimization over them is simply not feasible. As a solution, Schuth et al. [27] propose a method that samples from the allowed multileaved result lists and relaxes constraints when there is no exact solution. Consequently, there is no guarantee that this method does not introduce bias. Together, these two reasons show that the fidelity of OI does not imply fidelity of OM. It also shows that OM is computationally very costly.

\subsection{Probabilistic Multileaving}

Probabilistic Multileaving (PM) [28] is an extension of Probabilistic Interleaving (PI) [12], which was designed to solve the flaws of TDI Unlike the previous methods, PM considers every ranker as a distribution over documents, which is created by applying a soft-max to each of them. A multileaved result list is created by sampling a ranker with replacement at each iteration and sampling a document from the ranker that was selected. After the sampled document has been added, all rankers are renormalized to account for the removed document. During inference PM credits every ranker the expected number of clicked documents that were assigned to them. This is done by marginalizing over the possible ways the list could have been constructed by PM. A benefit of this approach is that it allows for comparisons on historical data $[12,13]$.

A big disadvantage of PM is that it allows any possible ranking to be shown, albeit not with uniform probabilities. This is a big deterrent for the usage of PM in operational settings. Furthermore, it also means that PM does not meet the considerateness requirement. On the other hand, PM does meet the fidelity requirements, the proof for this follows from the fact that every ranker is equally likely to add a document at each location in the ranking. Moreover, if multiple rankers want to place the same document somewhere they have to share the resulting credits. ${ }^{1}$ Similar to OM, PM becomes infeasible to compute for a large number of rankers $|\mathcal{R}|$; the number of assignments in the worst case is $|R|^{k}$. Fortunately, PM inference can be estimated by sampling assignments in a way that maintains fidelity $[22,28]$.

\subsection{Sample Only Scored Multileaving}

Sample-Scored-Only Multileaving (SOSM) was introduced by Brost et al. [2] in an attempt to create a more scalable multileaved comparison method. It is the only existing multileaved comparison method that does not have an interleaved comparison counterpart. SOSM attempts to increase sensitivity by ignoring all non-sampled documents during inference. Thus, at each impression a ranker receives credits according to how it ranks the documents that were sampled for the displayed multileaved result list of size $k$. The preferences at each impression are made binary before being added to the mean. SOSM creates multileaved result lists following the same procedure as TDM, a choice that seems arbitrary.

SOSM meets the considerateness requirements for the same reason TDM does. However, SOSM does not meet the fidelity requirement. We can prove this by providing an example where preferences are found under uncorrelated clicks. Consider the two documents $A$ and $B$ and the three rankers with the following three rankings:

$$
\mathbf{l}_{1}=A B, \quad \mathbf{l}_{2}=\mathbf{l}_{3}=B A .
$$

The first requirement of fidelity states that under uncorrelated clicks no preferences may be found in expectation. Uncorrelated clicks are unconditioned on document relevance (Equation 2); however, it is possible that they display position bias [32]. Thus the probability of a click at the first rank may be greater than at the second:

$$
P\left(\mathbf{c}_{1} \mid q\right)>P\left(\mathbf{c}_{2} \mid q\right) .
$$

Under position biased clicks the expected outcome for each possible multileaved result list is not zero. For instance, the following preferences are expected:

$$
\begin{aligned}
& E\left[P_{12} \mid \mathbf{m}=A B\right]>0, \\
& E\left[P_{12} \mid \mathbf{m}=B A\right]<0, \\
& E\left[P_{12} \mid \mathbf{m}=A B\right]=-E\left[P_{12} \mid \mathbf{m}=B A\right] .
\end{aligned}
$$

Since SOSM creates multileaved result lists following the TDM procedure the probability $P(\mathbf{m}=B A)$ is twice as high as $P(\mathbf{m}=A B)$.

${ }^{1}$ Brost et al. [2] proved that if the preferences at each impression are made binary the fidelity of PM is lost. 
Table 1: Overview of multileaved comparison methods and whether they meet the considerateness and fidelity requirements.

\begin{tabular}{lccc}
\hline & Considerateness & Fidelity & Source \\
\hline TDM & $\checkmark$ & & {$[27]$} \\
OM & $\checkmark$ & & {$[27]$} \\
PM & & $\checkmark$ & {$[28]$} \\
SOSM & $\checkmark$ & & {$[2]$} \\
PPM & $\checkmark$ & $\checkmark$ & this paper \\
\hline
\end{tabular}

As a consequence, the expected preference is biased against the first ranker:

$$
E\left[P_{12}\right]<0 .
$$

Hence, SOSM does not have fidelity. This outcome seems to stem from a disconnect between how multileaved results lists are created and how preferences are inferred.

To conclude this section, Table 1 provides an overview of our findings thus far, i.e., the theoretical requirements that each multileaved comparison method satisfies; we have also included PPM, the multileaved comparison method that we will introduce below.

\section{A NOVEL MULTILEAVED COMPARISON METHOD}

The previously described multileaved comparison methods are based around direct credit assignment, i.e., credit functions are based on single documents. In contrast, we introduce a method that estimates differences based on pairwise document preferences. We prove that this novel method is the only multileaved comparison method that meets the considerateness and fidelity requirements set out in Section 3.

The multileaved comparison method that we introduce is Pairwise Preference Multileaving (PPM). It infers pairwise preferences between documents from clicks and bases comparisons on the agreement of rankers with the inferred preferences. PPM is based on the assumption that a clicked document is preferred to: (a) all of the unclicked documents above it; (b) the next unclicked document. These assumptions are long-established [16] and form the basis of pairwise Learning to Rank (LTR) [15].

We write $\mathbf{c}_{r\left(d_{i}, \mathbf{m}\right)}$ for a click on document $d_{i}$ displayed in multileaved result list $\mathbf{m}$ at the rank $r\left(d_{i}, \mathbf{m}\right)$. For a document pair $\left(d_{i}, d_{j}\right)$, a click $\mathbf{c}_{r\left(d_{i}, \mathbf{m}\right)}$ infers a preference as follows:

$$
\begin{aligned}
& c_{r\left(d_{i}, \mathbf{m}\right)} \wedge \neg c_{r\left(d_{j}, \mathbf{m}\right)} \wedge\left(\exists i,\left(c_{i} \wedge r\left(d_{j}, \mathbf{m}\right)<i\right) \vee c_{r\left(d_{j}, \mathbf{m}\right)-1}\right) \\
& \Leftrightarrow d_{i}>_{c} d_{j} .
\end{aligned}
$$

In addition, the preference of a ranker $\mathbf{r}$ is denoted by $d_{i}>_{\mathbf{r}} d_{j}$. Pairwise preferences also form the basis for Preference-Based Balanced Interleaving (PBI) introduced by $\mathrm{He}$ et al. [11]. However, previous work has shown that PBI does not meet the fidelity requirements [13]. Therefore, we do not use PBI as a starting point for PPM. Instead, PPM is derived directly from the considerateness and fidelity requirements. Consequently, PPM constructs multileaved result lists inherently differently and its inference method has fidelity, in contrast with PBI.
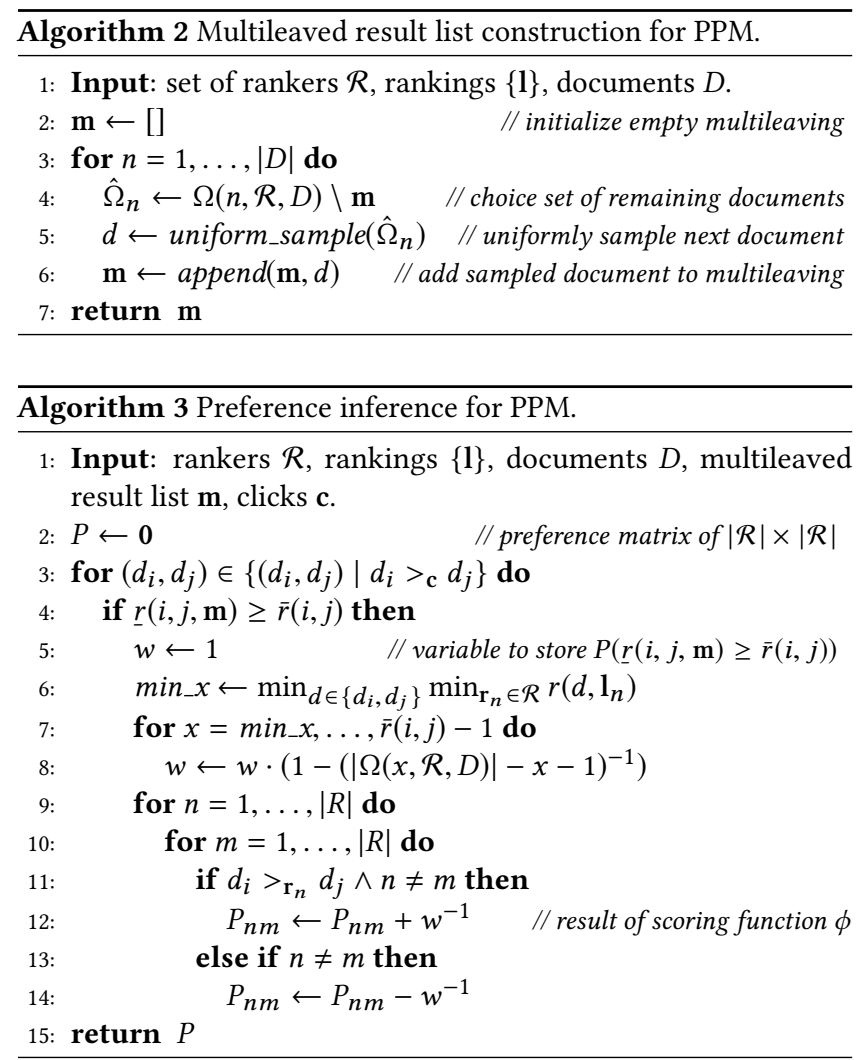

When constructing a multileaved result list $\mathbf{m}$ we want to be able to infer unbiased preferences while simultaneously being considerate. Thus, with the requirement for considerateness in mind we define a choice set as:

$$
\Omega(i, \mathcal{R}, D)=\left\{d \mid d \in D \wedge \exists \mathbf{r}_{j} \in \mathcal{R}, r\left(d, \mathbf{l}_{j}\right) \leq i\right\} .
$$

This definition is chosen so that any document in $\Omega(i, \mathcal{R}, D)$ can be placed at rank $i$ without breaking the obstruction requirement (Equation 1). The multileaving method of PPM is described in Algorithm 2. The approach is straightforward: at each rank $n$ the set of documents $\hat{\Omega}_{n}$ is determined (Line 4). This set of documents is $\Omega(n, \mathcal{R}, D)$ with the previously added documents removed to avoid document repetition. Then, the next document is sampled uniformly from $\hat{\Omega}_{n}$ (Line 5), thus every document in $\hat{\Omega}_{n}$ has a probability:

$$
\frac{1}{|\Omega(n, \mathcal{R}, D)|-n+1}
$$

of being placed at position $n$ (Line 6). Since $\hat{\Omega}_{n} \subseteq \Omega(n, \mathcal{R}, D)$ the resulting $\mathbf{m}$ is guaranteed to be considerate.

While the multileaved result list creation method used by PPM is simple, its preference inference method is more complicated as it has to meet the fidelity requirements. First, the preference found between a ranker $\mathbf{r}_{n}$ and $\mathbf{r}_{m}$ from a single interaction $\mathbf{c}$ is determined by:

$$
P_{n m}=\sum_{d_{i}>{ }_{c} d_{j}} \phi\left(d_{i}, d_{j}, \mathbf{r}_{n}, \mathbf{m}, \mathcal{R}\right)-\phi\left(d_{i}, d_{j}, \mathbf{r}_{m}, \mathbf{m}, \mathcal{R}\right),
$$


which sums over all document pairs $\left(d_{i}, d_{j}\right)$ where interaction c inferred a preference. Before the scoring function $\phi$ can be defined we introduce the following function:

$$
\bar{r}(i, j, \mathcal{R})=\max _{d \in\left\{d_{i}, d_{j}\right\}} \min _{\mathbf{r}_{n} \in \mathcal{R}} r\left(d, \mathbf{l}_{n}\right) .
$$

For succinctness we will note $\bar{r}(i, j)=\bar{r}(i, j, \mathcal{R})$. Here, $\bar{r}(i, j)$ provides the highest rank at which both documents $d_{i}$ and $d_{j}$ can appear in m. Position $\bar{r}(i, j)$ is important to the document pair $\left(d_{i}, d_{j}\right)$, since if both documents are in the remaining documents $\hat{\Omega}_{\bar{r}(i, j)}$, then the rest of the multileaved result list creation process is identical for both. To keep notation short we introduce:

$$
\underline{r}(i, j, \mathbf{m})=\min _{d \in\left\{d_{i}, d_{j}\right\}} r(d, \mathbf{m}) .
$$

Therefore, if $\underline{r}(i, j, \mathbf{m}) \geq \bar{r}(i, j)$ then both documents appear below $\bar{r}(i, j)$. This, in turn, means that both documents are equally likely to appear at any rank:

$$
\begin{aligned}
& \forall n, \quad P\left(\mathbf{m}_{n}=d_{i} \mid \underline{r}(i, j, \mathbf{m}) \geq \bar{r}(i, j)\right) \\
& \quad=P\left(\mathbf{m}_{n}=d_{j} \mid \underline{r}(i, j, \mathbf{m}) \geq \bar{r}(i, j)\right) .
\end{aligned}
$$

The scoring function $\phi$ is then defined as follows:

$$
\phi\left(d_{i}, d_{j}, \mathbf{r}, \mathbf{m}\right)= \begin{cases}0, & \underline{r}(i, j, \mathbf{m})<\bar{r}(i, j) \\ \frac{-1}{P(\underline{r}(i, j, \mathbf{m}) \geq \bar{r}(i, j))}, & d_{i}<_{\mathbf{r}} d_{j} \\ \frac{1}{P(\underline{r}(i, j, \mathbf{m}) \geq \bar{r}(i, j))}, & d_{i}>_{\mathbf{r}} d_{j},\end{cases}
$$

indicating that a zero score is given if one of the documents appears above $\bar{r}(i, j)$. Otherwise, the value of $\phi$ is positive or negative depending on whether the ranker $r$ agrees with the inferred preference between $d_{i}$ and $d_{j}$. Furthermore, this score is inversely weighed by the probability $P(r(i, j, \mathbf{m}) \geq \bar{r}(i, j))$. Therefore, pairs that are less likely to appear below their threshold $\bar{r}(i, j)$ result in a higher score than for more commonly occuring pairs. Algorithm 3 displays how the inference of PPM can be computed. The scoring function $\phi$ was carefully chosen to guarantee fidelity, the remainder of this section will sketch the proof for PPM meeting its requirements.

The two requirements for fidelity will be discussed in order:

Requirement 1 . The first fidelity requirement states that under uncorrelated clicks the expected outcome should be zero. Consider the expected preference:

$$
\begin{aligned}
E\left[P_{n m}\right]= & \sum_{d_{i}, d_{j}} \sum_{\mathbf{m}} P\left(d_{i}>_{c} d_{j} \mid \mathbf{m}\right) P(\mathbf{m}) \\
& \left(\phi\left(d_{i}, d_{j}, \mathbf{r}_{n}, \mathbf{m}\right)-\phi\left(d_{i}, d_{j}, \mathbf{r}_{m}, \mathbf{m}\right)\right) .
\end{aligned}
$$

To see that $E\left[P_{n m}\right]=0$ under uncorrelated clicks, take any multileaving $\mathbf{m}$ where $P(\mathbf{m})>0$ and $\phi\left(d_{i}, d_{j}, \mathbf{r}, \mathbf{m}\right) \neq 0$ with $\mathbf{m}_{x}=d_{i}$ and $\mathbf{m}_{y}=d_{j}$. Then there is always a multileaved result list $\mathbf{m}^{\prime}$ that is identical expect for swapping the two documents so that $\mathbf{m}_{x}^{\prime}=d_{j}$ and $\mathbf{m}_{y}^{\prime}=d_{i}$. The scoring function only gives non-zero values if both documents appear below the threshold $\underline{r}(i, j, \mathbf{m})<\bar{r}(i, j)$ (Equation 14). At this point the probability of each document appearing at any position is the same (Equation 13), thus the following holds:

$$
\begin{aligned}
P(\mathbf{m}) & =P\left(\mathbf{m}^{\prime}\right), \\
\phi\left(d_{i}, d_{j}, \mathbf{r}_{n}, \mathbf{m}\right) & =-\phi\left(d_{j}, d_{i}, \mathbf{r}_{n}, \mathbf{m}^{\prime}\right) .
\end{aligned}
$$

Finally, from the definition of uncorrelated clicks (Equation 2) the following holds:

$$
P\left(d_{i}>_{c} d_{j} \mid \mathbf{m}\right)=P\left(d_{j}>_{c} d_{i} \mid \mathbf{m}^{\prime}\right) .
$$

As a result, any document pair $\left(d_{i}, d_{j}\right)$ and multileaving $\mathbf{m}$ that affects the expected outcome is cancelled by the multileaving $\mathbf{m}^{\prime}$. Therefore, we can conclude that $E\left[P_{n m}\right]=0$ under uncorrelated clicks, and that PPM meets the first requirement of fidelity.

Requirement 2. The second fidelity requirement states that under correlated clicks a ranker that Pareto dominates all other rankers should win the multileaved comparison. Therefore, the expected value for a Pareto dominating ranker $r_{n}$ should be:

$$
\forall m, n \neq m \rightarrow E\left[P_{n m}\right]>0 .
$$

Take any other ranker $\mathbf{r}_{m}$ that is thus Pareto dominated by $\mathbf{r}_{n}$. The proof for the first requirement shows that $E\left[P_{n m}\right]$ is not affected by any pair of documents $d_{i}, d_{j}$ with the same relevance label. Furthermore, any pair on which $\mathbf{r}_{n}$ and $\mathbf{r}_{m}$ agree will not affect the expected outcome since:

$$
\begin{aligned}
& \left(d_{i}>_{\mathbf{r}_{n}} d_{j} \leftrightarrow d_{i}>_{\mathbf{r}_{m}} d_{j}\right) \Rightarrow \\
& \quad \phi\left(d_{i}, d_{j}, \mathbf{r}_{n}, \mathbf{m}\right)-\phi\left(d_{i}, d_{j}, \mathbf{r}_{m}, \mathbf{m}\right)=0 .
\end{aligned}
$$

Then, for any relevant document $d_{i}$, consider the set of documents that $\mathbf{r}_{n}$ incorrectly prefers over $d_{i}$ :

$$
A=\left\{d_{j} \mid \neg \operatorname{rel}\left(d_{j}\right) \wedge d_{j}>_{\mathbf{r}_{n}} d_{i}\right\}
$$

and the set of documents that $\mathbf{r}_{m}$ incorrectly prefers over $d_{i}$ and places higher than where $\mathbf{r}_{n}$ places $d_{i}$ :

$$
B=\left\{d_{j} \mid \neg \operatorname{rel}\left(d_{j}\right) \wedge d_{j}>_{\mathbf{r}_{m}} d_{i} \wedge r\left(d_{j}, \mathbf{l}_{m}\right)<r\left(d_{i}, \mathbf{l}_{n}\right)\right\} .
$$

Since $\mathbf{r}_{n}$ Pareto dominates $\mathbf{r}_{m}$, it has the same or fewer incorrect preferences: $|A| \leq|B|$. Furthermore, for any document $d_{j}$ in either $A$ or $B$ the threshold of the pair $d_{i}, d_{j}$ is the same:

$$
\forall d_{j} \in A \cup B, \bar{r}(i, j)=r\left(d_{i}, \mathbf{l}_{n}\right) .
$$

Therefore, all pairs with documents from $A$ and $B$ will only get a non-zero value from $\phi$ if they both appear at or below $r\left(d_{i}, 1_{n}\right)$. Then using Equation 13 and the Bayes rule we see:

$$
\begin{aligned}
\forall\left(d_{j}, d_{l}\right) \in A \cup B, & \frac{P\left(\mathbf{m}_{x}=d_{j}, \underline{r}(i, j, \mathbf{m}) \geq \bar{r}(i, j, \mathcal{R})\right)}{P(\underline{r}(i, j, \mathbf{m}) \geq \bar{r}(i, j, \mathcal{R}))} \\
& =\frac{P\left(\mathbf{m}_{x}=d_{l}, \underline{r}(i, l, \mathbf{m}) \geq \bar{r}(i, l, \mathcal{R})\right)}{P(\underline{r}(i, l, \mathbf{m}) \geq \bar{r}(i, l, \mathcal{R}))} .
\end{aligned}
$$

Similarly, the reweighing of $\phi$ ensures that every pair in $A$ and $B$ contributes the same to the expected outcome. Thus, if both rankers rank $d_{i}$ at the same position the following sum:

$$
\begin{aligned}
& \sum_{d_{j} \in A \cup B} \sum_{\mathbf{m}} P(\mathbf{m}) \cdot \\
& \quad\left[P\left(d_{i}>_{c} d_{j} \mid \mathbf{m}\right)\left(\phi\left(d_{i}, d_{j}, \mathbf{r}_{n}, \mathbf{m}\right)-\phi\left(d_{i}, d_{j}, \mathbf{r}_{m}, \mathbf{m}\right)\right)\right. \\
& \left.\quad+P\left(d_{j}>_{c} d_{i} \mid \mathbf{m}\right)\left(\phi\left(d_{j}, d_{i}, \mathbf{r}_{n}, \mathbf{m}\right)-\phi\left(d_{j}, d_{i}, \mathbf{r}_{m}, \mathbf{m}\right)\right)\right]
\end{aligned}
$$

will be zero if $|A|=|B|$ and positive if $|A|<|B|$ under correlated clicks. Moreover, since $\mathbf{r}_{n}$ Pareto dominates $\mathbf{r}_{m}$, there will be at least one document $d_{j}$ where:

$$
\exists d_{i}, \exists d_{j}, \operatorname{rel}\left(d_{i}\right) \wedge \neg \operatorname{rel}\left(d_{j}\right) \wedge r\left(d_{i}, 1_{n}\right)=r\left(d_{j}, 1_{m}\right) .
$$


This means that the expected outcome (Equation 15) will always be positive under correlated clicks, i.e., $E\left[P_{n m}\right]>0$, for a Pareto dominating ranker $\mathbf{r}_{n}$ and any other ranker $\mathbf{r}_{m}$.

In summary, we have introduced a new multileaved comparison method, PPM, which we have shown to be considerate and to have fidelity. We further note that PPM has polynomial complexity: to calculate $P(\underline{r}(i, j, \mathbf{m}) \geq \bar{r}(i, j))$ only the size of the choice sets $\Omega$ and the first positions at which $d_{i}$ and $d_{j}$ occur in $\Omega$ have to be known.

\section{EXPERIMENTS}

In order to answer Research Question RQ2 posed in Section 1 several experiments were performed to evaluate the sensitivity of PPM. The methodology of evaluation follows previous work on interleaved and multileaved comparison methods [2, 12, 13, 27, 28] and is completely reproducible. ${ }^{2}$

\subsection{Ranker selection and comparisons}

In order to make fair comparisons between rankers, we will use the Online Learning to Rank (OLTR) datasets described in Section 6.2. From the feature representations in these datasets a handpicked set of features was taken and used as ranking models. To match the real-world scenario as best as possible this selection consists of features which are known to perform well as relevance signals independently. This selection includes but is not limited to: BM25, LMIR.JM, Sitemap, PageRank, HITS and TF.IDF.

Then the ground-truth comparisons between the rankers are based on their NDCG scores computed on a held-out test set, resulting in a binary preference matrix $P_{n m}$ for all ranker pairs $\left(\mathbf{r}_{n}, \mathbf{r}_{m}\right)$ :

$$
P_{n m}=N D C G\left(\mathbf{r}_{n}\right)-N D C G\left(\mathbf{r}_{m}\right) .
$$

The metric by which multileaved comparison methods are compared is the binary error, $E_{b i n}[2,27,28]$. Let $\hat{P}_{n m}$ be the preference inferred by a multileaved comparison method; then the error is:

$$
E_{\text {bin }}=\frac{\sum_{n, m \in \mathcal{R} \wedge n \neq m} \operatorname{sgn}\left(\hat{P}_{n m}\right) \neq \operatorname{sgn}\left(P_{n m}\right)}{|\mathcal{R}| \times(|\mathcal{R}|-1)} .
$$

\subsection{Datasets}

Our experiments are performed over ten publicly available OLTR datasets with varying sizes and representing different search tasks. Each dataset consists of a set of queries and a set of corresponding documents for every query. While queries are represented only by their identifiers, feature representations and relevance labels are available for every document-query pair. Relevance labels are graded differently by the datasets depending on the task they model, for instance, navigational datasets have binary labels for not relevant (0), and relevant (1), whereas most informational tasks have labels ranging from not relevant (0), to perfect relevancy (5). Every dataset consists of five folds, each dividing the dataset in different training, validation and test partitions.

The first publicly available Learning to Rank datasets are distributed as LETOR 3.0 and 4.0 [21]; they use representations of 45 , 46, or 64 features encoding ranking models such as TF.IDF, BM25, Language Modelling, PageRank, and HITS on different parts of the documents. The datasets in LETOR are divided by their tasks, most of which come from the TREC Web Tracks between 2003 and

\footnotetext{
${ }^{2}$ https://github.com/HarrieO/PairwisePreferenceMultileave
}

Table 2: Instantiations of Cascading Click Models [10] as

\begin{tabular}{|c|c|c|c|c|c|c|c|c|c|c|}
\hline \multirow[b]{2}{*}{$R$} & \multicolumn{5}{|c|}{$P($ click $=1 \mid R)$} & \multicolumn{5}{|c|}{$P($ stop $=1 \mid R)$} \\
\hline & 0 & 1 & 2 & 3 & 4 & 0 & 1 & 2 & 3 & 4 \\
\hline perf & 0.0 & 0.2 & 0.4 & 0.8 & 1.0 & 0.0 & 0.0 & 0.0 & 0.0 & 0.0 \\
\hline nav & 0.05 & 0.3 & 0.5 & 0.7 & 0.95 & 0.2 & 0.3 & 0.5 & 0.7 & 0.9 \\
\hline inf & 0.4 & 0.6 & 0.7 & 0.8 & 0.9 & 0.1 & 0.2 & 0.3 & 0.4 & 0.5 \\
\hline
\end{tabular}
used for simulating user behaviour in experiments.

2008 [7, 8]. HP2003, HP2004, NP2003, NP2004, TD2003 and TD2004 each contain between 50 and 150 queries and 1,000 judged documents per query and use binary relevance labels. Due to their similarity we report average results over these six datasets noted as LETOR 3.0. The OHSUMED dataset is based on the query log of the search engine on the MedLine abstract database, and contains 106 queries. The last two datasets, MQ2007 and MQ2008, were based on the Million Query Track [1] and consist of 1,700 and 800 queries, respectively, but have far fewer assessed documents per query.

The MLSR-WEB10K dataset [23] consists of 10,000 queries obtained from a retired labelling set of a commercial web search engine. The datasets uses 136 features to represent its documents, each query has around 125 assessed documents.

Finally, we note there are more OLTR datasets available [3, 9], but there is no public information about their feature representations. Therefore, they are unfit for our evaluation as no selection of well performing ranking features can be made.

\subsection{Simulating user behavior}

While experiments using real users are preferred [4, 6, 18, 31], most researchers do not have access to search engines. As a result the most common way of comparing online evaluation methods is by using simulated user behaviour [2, 12, 13, 27, 28]. Such simulated experiments show the performance of multileaved comparison methods when user behaviour adheres to a few simple assumptions.

Our experiments follow the precedent set by previous work on online evaluation: First, a user issues a query simulated by uniformly sampling a query from the static dataset. Subsequently, the multileaved comparison method constructs the multileaved result list of documents to display. The behavior of the user after receiving this list is simulated using a cascade click model $[5,10]$. This model assumes a user to examine documents in their displayed order. For each document that is considered the user decides whether it warrants a click, which is modeled as the conditional probability $P($ click $=1 \mid R)$ where $R$ is the relevance label provided by the dataset. Accordingly, cascade click model instantiations increase the probability of a click with the degree of the relevance label. After the user has clicked on a document their information need may be satisfied; otherwise they continue considering the remaining documents. The probability of the user not examining more documents after clicking is modeled as $P($ stop $=1 \mid R)$, where it is more likely that the user is satisfied from a very relevant document. At each impression we display $k=10$ documents to the user.

Table 2 lists the three instantiations of cascade click models that we use for this paper. The first models a perfect user (perf) who considers every document and clicks on all relevant documents and nothing else. Secondly, the navigational instantiation (nav) models a user performing a navigational task who is mostly looking 
for a single highly relevant document. Finally, the informational instantiation (inf) models a user without a very specific information need who typically clicks on multiple documents. These three models have increasing levels of noise, as the behavior of each depends less on the relevance labels of the displayed documents.

\subsection{Experimental runs}

Each experimental run consists of applying a multileaved comparison method to a sequence of $T=10,000$ simulated user impressions. To see the effect of the number of rankers in a comparison, our runs consider $|\mathcal{R}|=5,|\mathcal{R}|=15$, and $|\mathcal{R}|=40$. However only the MSLR dataset contains $|\mathcal{R}|=40$ rankers. Every run is repeated for every click model to see how different behaviours affect performance. For statistical significance every run is repeated 25 times per fold, which means that 125 runs are conducted for every dataset and click model pair. Since our evaluation covers five multileaved comparison methods, we generate over 393 million impressions in total. We test for statistical significant differences using a two tailed t-test. Note that the results reported on the LETOR 3.0 data are averaged over six datasets and thus span 750 runs per datapoint.

The parameters of the baselines are selected based on previous work on the same datasets; for OM the sample size $\eta=10$ was chosen as reported by Schuth et al. [27]; for PM the degree $\tau=3.0$ was chosen according to Hofmann et al. [12] and the sample size $\eta=10,000$ in accordance with Schuth et al. [28].

\section{RESULTS AND ANALYSIS}

We answer Research Question RQ2 by evaluating the sensitivity of PPM based on the results of the experiments detailed in Section 6 .

The results of the experiments with a smaller number of rankers: $|\mathcal{R}|=5$ are displayed in Table 3 . Here we see that after 10,000 impressions PPM has a significantly lower error on many datasets and at all levels of interaction noise. Furthermore, for $|\mathcal{R}|=5$ there are no significant losses in performance under any circumstances.

When $|\mathcal{R}|=15$ as displayed in Table 4, we see a single case where PPM performs worse than a previous method: on MQ2007 under the perfect click model SOSM performs significantly better than PPM. However, on the same dataset PPM performs significantly better under the informational click model. Furthermore, there are more significant improvements for $|\mathcal{R}|=15$ than when the number of rankers is the smaller $|\mathcal{R}|=5$.

Finally, when the number of rankers in the comparison is increased to $|\mathcal{R}|=40$ as displayed in Table 5, PPM still provides significant improvements.

We conclude that PPM always provides a performance that is at least as good as any existing method. Moreover, PPM is robust to noise as we see more significant improvements under click-models with increased noise. Furthermore, since improvements are found with the number of rankers $|\mathcal{R}|$ varying from 5 to 40 , we conclude that PPM is scalable in the comparison size. Additionally, the dataset type seems to affect the relative performance of the methods. For instance, on LETOR 3.0 little significant differences are found, whereas the MSLR dataset displays the most significant improvements. This suggests that on more artificial data, i.e., the smaller datasets simulating navigational tasks, the differences are
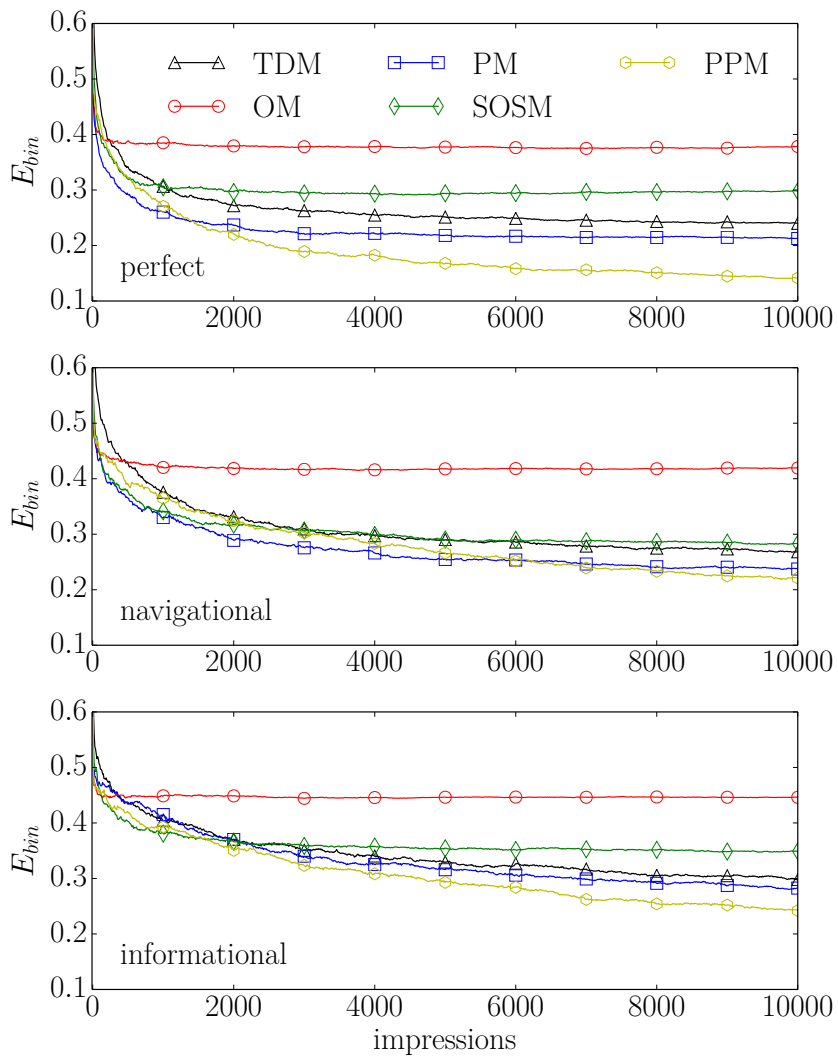

Figure 1: The binary error of different multileaved comparison methods on comparisons of $|\mathcal{R}|=15$ rankers on the MSLR-WEB10k dataset.

fewer, while on the other hand on large commercial data the preference for PPM increases further. Lastly, Figure 1 displays the binary error of all multileaved comparison methods on the MSLR dataset over 10,000 impressions. Under the perfect click model we see that all of the previous methods display converging behavior around 3,000 impressions. In contrast, the error of PPM continues to drop throughout the experiment. The fact that the existing methods converge at a certain level of error in the absence of click-noise is indicative that they are lacking in sensitivity.

Overall, our results show that PPM reaches a lower level of error than previous methods seem to be capable of. This feat can be observed on a diverse set of datasets, various levels of interaction noise and for different comparison sizes. To answer Research Question RQ2: from our results we conclude that PPM is more sensitive than any existing multileaved comparison method.

\section{CONCLUSION}

In this paper we have examined multileaved comparison methods for evaluating ranking models online.

We have presented a new multileaved comparison method, Pairwise Preference Multileaving (PPM), that is more sensitive to user preferences than existing methods. Additionally, we have proposed a theoretical framework for assessing multileaved comparison methods, with considerateness and fidelity as the two key requirements. 
Table 3: The binary error $E_{\text {bin }}$ of all multileaved comparison methods after 10,000 impressions on comparisons of $|\mathcal{R}|=5$ rankers. Average per dataset and click model; standard deviation in brackets. The best performance per click model and dataset is noted in bold, statistically significant improvements of PPM are noted by $\mathbf{\nabla}_{(p<0.01) \text { and }}^{\nabla}(p<0.05)$ and losses by $\Delta$ and ${ }^{\triangle}$ respectively or $\circ$ for no difference, per baseline.

\begin{tabular}{|c|c|c|c|c|c|}
\hline & TDM & OM & PM & SOSM & PPM \\
\hline \multicolumn{6}{|c|}{ perfect } \\
\hline LETOR 3.0 & $0.16(0.13)$ & 0.14 & $0.15(0.15)$ & $0.16(0.15)$ & $0.14(0.13) \circ \bigcirc ০ ০$ \\
\hline MQ2007 & $0.19(0.16)$ & $0.22(0.18)$ & $0.16(0.14)$ & 0.18 & $0.16(0.14) \circ \vee \circ \bigcirc$ \\
\hline MQ2008 & $0.15(0.12)$ & $0.19(0.14)$ & $0.16(0.12)$ & $0.18(0.15)$ & $0.14(0.12) \circ \vee \circ \nabla$ \\
\hline MSLR-WEB10k & $0.23(0.13)$ & $0.27(0.17)$ & $0.20(0.14)$ & $0.25(0.18)$ & $0.14(0.13) \nabla \nabla V$ \\
\hline OHSUMED & $0.14(0.12)$ & $0.19(0.15)$ & $0.11(0.09)$ & $0.11(0.10)$ & $0.11(0.10) \vee \nabla \circ \diamond$ \\
\hline \multicolumn{6}{|c|}{ navigational } \\
\hline LETOR 3.0 & $0.16(0.13)$ & 0.15 & $0.15(0.14)$ & $0.17(0.15)$ & $0.16(0.14) \circ \circ \circ \circ$ \\
\hline MQ2007 & $0.21(0.17)$ & $0.33(0.21)$ & $0.18(0.12)$ & $0.29(0.23)$ & $0.17(0.14) \circ \nabla \circ \nabla$ \\
\hline MQ2008 & $0.17(0.14)$ & $0.21(0.20)$ & $0.17(0.15)$ & $0.23(0.18)$ & $0.15(0.13) \circ \checkmark \circ \nabla$ \\
\hline MSLR-WEB10k & $0.24(0.14)$ & $0.32(0.20)$ & $0.24(0.17)$ & $0.31(0.19)$ & $0.20_{(0.15)} \nabla \nabla \nabla \nabla$ \\
\hline OHSUMED & $0.12(0.11)$ & $0.27(0.19)$ & $0.14(0.12)$ & $0.23(0.17)$ & $0.13(0.12) \circ \mathbf{\nabla} \circ \mathbf{\nabla}$ \\
\hline \multicolumn{6}{|c|}{ informational } \\
\hline LETOR 3.0 & $0.16(0.14)$ & $0.22(0.19)$ & $0.14(0.11)$ & $0.17(0.15)$ & $0.15(0.13) \circ \mathbf{V} \circ$ \\
\hline MQ2007 & $0.23(0.15)$ & $0.41(0.26)$ & $0.23(0.15)$ & $0.37(0.23)$ & $0.17_{(0.16)} \nabla \nabla V$ \\
\hline MQ2008 & $0.18(0.13)$ & $0.28(0.19)$ & $0.18(0.16)$ & $0.23(0.18)$ & $0.17(0.14) \circ \vee \circ \checkmark$ \\
\hline MSLR-WEB10k & $0.27(0.18)$ & $0.42(0.23)$ & $0.24(0.17)$ & $0.36(0.20)$ & $0.19(0.17) \vee \nabla \nabla \nabla$ \\
\hline OHSUMED & $0.13(0.10)$ & $0.37(0.24)$ & $0.12(0.11)$ & $0.27(0.21)$ & $0.12(0.10) \circ \checkmark \circ \checkmark$ \\
\hline
\end{tabular}

Table 4: The binary error $E_{\text {bin }}$ after 10,000 impressions on comparisons of $|\mathcal{R}|=15$ rankers. Notation is identical to Table 3.

\begin{tabular}{|c|c|c|c|c|c|}
\hline & TDM & OM & PM & SOSM & PPM \\
\hline \multicolumn{6}{|c|}{ perfect } \\
\hline LETOR 3.0 & $0.16(0.07)$ & $0.14(0.08)$ & $0.15(0.07)$ & $0.17(0.08)$ & $0.16(0.08) \circ \circ \circ \circ$ \\
\hline MQ2007 & $0.20(0.07)$ & $0.25(0.09)$ & $0.18(0.06)$ & $0.15(0.07)$ & $0.19(0.07) \bigcirc \mathbf{\nabla}$ \\
\hline MQ2008 & $0.16(0.05)$ & $0.17(0.05)$ & $0.16(0.05)$ & $0.15(0.07)$ & $0.15(0.06) \bigcirc \vee \bigcirc ০$ \\
\hline MSLR-WEB10k & $0.24(0.07)$ & $0.38(0.11)$ & $0.21(0.06)$ & $0.30(0.08)$ & $0.14(0.05)\rangle \nabla \nabla$ \\
\hline OHSUMED & $0.14(0.03)$ & $0.18(0.05)$ & $0.13(0.03)$ & $0.13(0.03)$ & $0.11_{(0.03)} \nabla \nabla \nabla$ \\
\hline \multicolumn{6}{|c|}{ navigational } \\
\hline LETOR 3.0 & $0.16(0.08)$ & $0.16(0.09)$ & 0.15 ( 0.08) & $0.17_{(0.08)}$ & $0.17(0.08) \quad \circ \circ 0 \circ$ \\
\hline MQ2007 & $0.24(0.07)$ & $0.33(0.11)$ & $0.20(0.07)$ & $0.22(0.08)$ & $0.21(0.08) \quad \boldsymbol{\nabla} \circ \circ$ \\
\hline MQ2008 & $0.19(0.05)$ & $0.21(0.07)$ & $0.16(0.05)$ & $0.18(0.06)$ & $0.16(0.06) \checkmark \nabla \circ \nabla$ \\
\hline MSLR-WEB10k & $0.27(0.07)$ & $0.42(0.12)$ & $0.24(0.06)$ & $0.28(0.09)$ & $0.22(0.08) \vee \nabla \circ \nabla$ \\
\hline OHSUMED & $0.14(0.04)$ & $0.25(0.07)$ & $0.13(0.03)$ & $0.18(0.06)$ & $0.13(0.04) \circ \checkmark \circ$ \\
\hline \multicolumn{6}{|c|}{ informational } \\
\hline LETOR 3.0 & $0.18(0.07)$ & $0.20(0.11)$ & $0.17(0.08)$ & $0.16(0.08)$ & ०.18)(0.08) ○ \\
\hline MQ2007 & $0.28(0.07)$ & $0.42(0.14)$ & $0.26(0.08)$ & $0.28(0.11)$ & $0.21_{(0.08)} \nabla \nabla \nabla$ \\
\hline MQ2008 & $0.23(0.06)$ & $0.26(0.11)$ & $0.18(0.06)$ & $0.20(0.06)$ & $0.15(0.06) \nabla \nabla \nabla \nabla$ \\
\hline MSLR-WEB10k & $0.30(0.09)$ & $0.45(0.12)$ & $0.28(0.08)$ & $0.35(0.11)$ & $0.24(0.08) \nabla \nabla V \nabla$ \\
\hline OHSUMED & $0.15(0.03)$ & $0.42(0.09)$ & $0.13(0.03)$ & $0.25(0.06)$ & $0.13(0.04) \vee \nabla \circ \nabla$ \\
\hline
\end{tabular}

We have shown that no method published prior to PPM has fidelity without lacking considerateness. In other words, prior to PPM no multileaved comparison method has been able to infer correct preferences without degrading the search experience of the user. In contrast, we prove that PPM has both considerateness and fidelity, thus it is guaranteed to correctly identify a Pareto dominating ranker without altering the search experience considerably. Furthermore, our experimental results spanning ten datasets show that PPM is more sensitive than existing methods, meaning that it can reach a lower level of error than any previous method. Moreover, our experiments show that the most significant improvements are obtained on the more complex datasets, i.e., larger datasets with more grades of relevance. Additionally, similar improvements are 
Table 5: The binary error $E_{b i n}$ of all multileaved comparison methods after 10,000 impressions on comparisons of $|\mathcal{R}|=40$ rankers. Averaged over the MSLR-WEB10k, notation is identical to Table 3.

\begin{tabular}{|c|c|c|c|c|c|}
\hline & TDM & OM & PM & SOSM & PPM \\
\hline perfect & $0.26(0.03)$ & $0.43(0.02)$ & $0.23(0.02)$ & $0.31(0.02)$ & $0.18(0.04) \backslash \nabla \nabla \nabla$ \\
\hline navigational & $0.31(0.03)$ & $0.44(0.01)$ & $0.25(0.03)$ & 0.23 & $0.24(0.05) \quad \nabla \nabla \nabla \circ$ \\
\hline informational & $0.37(0.04)$ & $0.47(0.01)$ & $0.30(0.05)$ & $0.34(0.05)$ & $0.27_{(0.06)} \nabla \nabla \nabla$ \\
\hline
\end{tabular}

observed under different levels of noise and numbers of rankers in the comparison, indicating that PPM is robust to interaction noise and scalable to large comparisons. As an extra benefit, the computational complexity of PPM is polynomial and, unlike previous methods, does not depend on sampling or approximations.

The theoretical framework that we have introduced allows future research into multileaved comparison methods to guarantee improvements that generalize better than empirical results alone. In turn, properties like considerateness can further stimulate the adoption of multileaved comparison methods in production environments; future work with real-world users may yield further insights into the effectiveness of the multileaving paradigm. Rich interaction data enables the introduction of multileaved comparison methods that consider more than just clicks, as has been done for interleaving methods [18]. These methods could be extended to consider other signals such as dwell-time or the order of clicks in an impression, etc.

Furthermore, the field of OLTR has depended on online evaluation from its inception [30]. The introduction of multileaving and following novel multileaved comparison methods brought substantial improvements to both fields [22, 29]. Similarly, PPM and any future extensions are likely to benefit the OLTR field too.

Finally, while the theoretical and empirical improvements of PPM are convincing, future work should investigate whether the sensitivity can be made even stronger. For instance, it is possible to have clicks from which no preferences between rankers can be inferred. Can we devise a method that avoids such situations as much as possible without introducing any form of bias, thus increasing the sensitivity even further while maintaining theoretical guarantees?

Acknowledgments. This research was supported by Ahold Delhaize, Amsterdam Data Science, the Bloomberg Research Grant program, the Criteo Faculty Research Award program, the Dutch national program COMMIT, Elsevier, the European Community's Seventh Framework Programme (FP7/2007-2013) under grant agreement nr 312827 (VOX-Pol), the Microsoft Research Ph.D. program, the Netherlands Institute for Sound and Vision, the Netherlands Organisation for Scientific Research (NWO) under project nrs 612.001.116, HOR-11-10, CI-14-25, 652.002.001, 612.001.551, 652.001.003, and Yandex. All content represents the opinion of the authors, which is not necessarily shared or endorsed by their respective employers and/or sponsors.

\section{REFERENCES}

[1] J. Allan, B. Carterette, J. A. Aslam, V. Pavlu, B. Dachev, and E. Kanoulas. Million query track 2007 overview. In TREC. NIST, 2007.

[2] B. Brost, I. J. Cox, Y. Seldin, and C. Lioma. An improved multileaving algorithm for online ranker evaluation. In SIGIR, pages 745-748. ACM, 2016

[3] O. Chapelle and Y. Chang. Yahoo! Learning to Rank Challenge Overview. fournal of Machine Learning Research, 14:1-24, 2011.
[4] O. Chapelle, T. Joachims, F. Radlinski, and Y. Yue. Large-scale validation and analysis of interleaved search evaluation. TOIS, 30(1):Article 6, 2012.

[5] A. Chuklin, I. Markov, and M. de Rijke. Click Models for Web Search. Morgan \& Claypool Publishers, 2015.

[6] A. Chuklin, A. Schuth, K. Zhou, and M. de Rijke. A comparative analysis of interleaving methods for aggregated search. TOIS, 33(2):Article 5, 2015.

[7] C. L. Clarke, N. Craswell, and I. Soboroff. Overview of the TREC 2009 Web Track. In TREC. NIST, 2009.

[8] N. Craswell, D. Hawking, R. Wilkinson, and M. Wu. Overview of the TREC 2003 Web Track. In TREC. NIST, 2003.

[9] D. Dato, C. Lucchese, F. M. Nardini, S. Orlando, R. Perego, N. Tonellotto, and R. Venturini. Fast ranking with additive ensembles of oblivious and non-oblivious regression trees. TOIS, 35(2):15, 2016.

[10] F. Guo, C. Liu, and Y. M. Wang. Efficient multiple-click models in web search. In WSDM, pages 124-131. ACM, 2009.

[11] J. He, C. Zhai, and X. Li. Evaluation of methods for relative comparison of retrieval systems based on clickthroughs. In CIKM, pages 2029-2032. ACM, 2009.

[12] K. Hofmann, S. Whiteson, and M. de Rijke. A probabilistic method for inferring preferences from clicks. In CIKM, pages 249-258. ACM, 2011.

[13] K. Hofmann, S. Whiteson, and M. de Rijke. Fidelity, soundness, and efficiency of interleaved comparison methods. TOIS, 31(4):17, 2013.

[14] K. Hofmann, L. Li, and F. Radlinski. Online evaluation for information retrieval. Foundations and Trends in Information Retrieval, 10(1):1-117, 2016.

[15] T. Joachims. Optimizing search engines using clickthrough data. In $K D D$, pages 133-142. ACM, 2002

[16] T. Joachims. Unbiased evaluation of retrieval quality using clickthrough data. In SIGIR Workshop on Mathematical/Formal Methods in Information Retrieval, volume 354, 2002.

[17] T. Joachims. Evaluating retrieval performance using clickthrough data. In Text Mining. Physica/Springer, 2003.

[18] E. Kharitonov, C. Macdonald, P. Serdyukov, and I. Ounis. Generalized team draft interleaving. In CIKM, pages 773-782. ACM, 2015.

[19] R. Kohavi, R. Longbotham, D. Sommerfield, and R. M. Henne. Controlled experiments on the web: survey and practical guide. Data Mining and Knowledge Discovery, 18(1):140-181, 2009.

[20] R. Kohavi, A. Deng, B. Frasca, T. Walker, Y. Xu, and N. Pohlmann. Online controlled experiments at large scale. In KDD, pages 1168-1176. ACM, 2013.

[21] T.-Y. Liu, J. Xu, T. Qin, W. Xiong, and H. Li. LETOR: Benchmark dataset for research on learning to rank for information retrieval. In LR4IR '07, 2007.

[22] H. Oosterhuis, A. Schuth, and M. de Rijke. Probabilistic multileave gradient descent. In ECIR, pages 661-668. Springer, 2016.

[23] T. Oin and T. Liu. Introducing LETOR 4.0 datasets. CoRR, abs/1306.2597, 2013.

[24] F. Radlinski and N. Craswell. Optimized interleaving for online retrieval evaluation. In WSDM, pages 245-254. ACM, 2013.

[25] F. Radlinski, M. Kurup, and T. Joachims. How does clickthrough data reflect retrieval quality? In CIKM, pages 43-52. ACM, 2008.

[26] M. Sanderson. Test collection based evaluation of information retrieval systems. Foundations and Trends in Information Retrieval, 4(4):247-375, 2010.

[27] A. Schuth, F. Sietsma, S. Whiteson, D. Lefortier, and M. de Rijke. Multileaved comparisons for fast online evaluation. In CIKM, pages 71-80. ACM, 2014

[28] A. Schuth, R.-J. Bruintjes, F. Büttner, J. van Doorn, et al. Probabilistic multileave for online retrieval evaluation. In SIGIR, pages 955-958. ACM, 2015.

[29] A. Schuth, H. Oosterhuis, S. Whiteson, and M. de Rijke. Multileave gradient descent for fast online learning to rank. In WSDM, pages 457-466. ACM, 2016.

[30] Y. Yue and T. Joachims. Interactively optimizing information retrieval systems as a dueling bandits problem. In ICML' '09, 2009.

[31] Y. Yue, Y. Gao, O. Chapelle, Y. Zhang, and T. Joachims. Learning more powerful test statistics for click-based retrieval evaluation. In SIGIR, pages 507-514. ACM, 2010 .

[32] Y. Yue, R. Patel, and H. Roehrig. Beyond position bias: Examining result attractiveness as a source of presentation bias in clickthrough data. In $W W W$, pages 1011-1018. ACM, 2010. 